\title{
Ultrafast extreme ultraviolet photoemission without space charge
}

\author{
Peng Zhao ${ }^{l}$, Christopher Corder ${ }^{l}$, Jin Bakalis ${ }^{l}$,Xinlong $\mathrm{Li}^{l}$, Matthew D. \\ Kershis $^{1,2}$, Amanda R. Muraca ${ }^{l}$ Michael $G$. White ${ }^{1,2, *}$, and Thomas K. Allison ${ }^{l, *}$ \\ ${ }^{1}$ Stony Brook University, Stony Brook, NY, 11794,USA \\ ${ }^{2}$ Brookhaven National Lab, Brookhaven Avenue, Upton, NY, 11973, USA
}

\begin{abstract}
We present photoelectron spectroscopy experiments using an $88 \mathrm{MHz}$ cavityenhanced high-harmonic source operating from 8 to $40 \mathrm{eV}$. Nanoampere space-charge free sample photo currents enable us to record time-resolved photoelectron spectra from weakly excited samples.
\end{abstract}

\section{Introduction}

Extreme ultraviolet (XUV) light sources based on high harmonic generation (HHG) have been widely applied to time resolved photoemission spectroscopy (TRPES) on surfaces. Compared to synchrotrons experiments, TRPES experiments using HHG are drastically limited due to "space-charge effects" [1]. Due to the high peak powers required to drive the $\mathrm{HHG}$ process, HHG is typically restricted to laser systems with $<100 \mathrm{kHz}$ repetition rates. In order to achieve the same photocurrent as synchrotron experiments, the electrons must be concentrated. The coulomb repulsion between the electrons can distort the photoelectron spectrum on the eV scale. Pump pulses can also produce electrons due to multiphoton ionization contributing to the space charge problem. Time-resolved photoelectron spectroscopy experiments using HHG have to compromise on the applied photon flux, focused spot size, resolution, fidelity of the signal, or some combination thereof [2].

In this article we demonstrate the application of a tunable cavity-enhanced high-harmonic generation (CEHHG) light source to surface photoemission. By performing experiments with high flux at $88 \mathrm{MHz}$ repetition rate, nanoamperes of sample current can be generated from a sub-100 micron XUV spot with space charge effects less than $10 \mathrm{meV}$. As we show, this enables time-resolved photoelectron spectroscopy in a qualitatively different regime of sensitivity and pump-fluence than space-charge limited systems.

\section{Instrument}

Our instrument is described in detail in Ref. [3,4]. A home-built $80 \mathrm{~W}, 155 \mathrm{fs}$ frequency comb

*Corresponding author: Thomas.Allison@,stonybrook.edu, Michael.G.White@stonybrook.edu 
laser [5] is passively amplified in an enhancement cavity to 5-11 $\mathrm{kW}$. Harmonics are generated at the intracavity focus and reflected from a sapphire wafer placed a Brewster's angle. The outcoupled harmonics are selected by a home-built pulse preserving monochromator similar to the design of Frassetto et al. [6], and then are 1:1 imaged to a sample in our surface science chamber. Fig. 1 (a) shows the typical photon flux at the sample versus photon energy for argon, krypton, and xenon generating gas. By changing the generating gas, a flux of more than 1011 photons per second is delivered to the sample over a broad energy range. These fluxes are within one order of magnitude of what is available from many state-of-the-art synchrotron beamlines dedicated to ARPES. Critically, since at $88 \mathrm{MHz}, 10^{11}$ photons/s corresponds to only a few thousand photons/pulse - also comparable to synchrotrons, all of this flux is usable for high-resolution photoemission experiments.

\section{Time resolved photoemission experiment}

We demonstrate the absence of space-charge effects in a measurement of laser assisted photoemission (LAPE) [7] from Au (111) surface. Figure 1 (b) shows two photoelectron spectra near the Fermi edge of the Au (111) on a logarithmic scale, one with and another without a parallel polarized $1.035 \mu \mathrm{m}$ wavelength pump laser. The spectra were taken with 3 nA of sample current, or approximately 215 electrons per pulse. For the black curve with the laser off, a precipitous drop of four orders of magnitude is observed. For the red curve with the pump laser on, a sideband of the surface state peak at $24.8 \mathrm{eV}$ is observed $1.2 \mathrm{eV}$ higher at $26 \mathrm{eV}$, as expected due to LAPE. No energy broadening or shifting is seen in either of the two curved, because the high data rate enables the experiment to be performed under the low pump peak intensity of $1.3 \times 109 \mathrm{~W} / \mathrm{cm}^{2}$. At this low intensity, the multiphoton
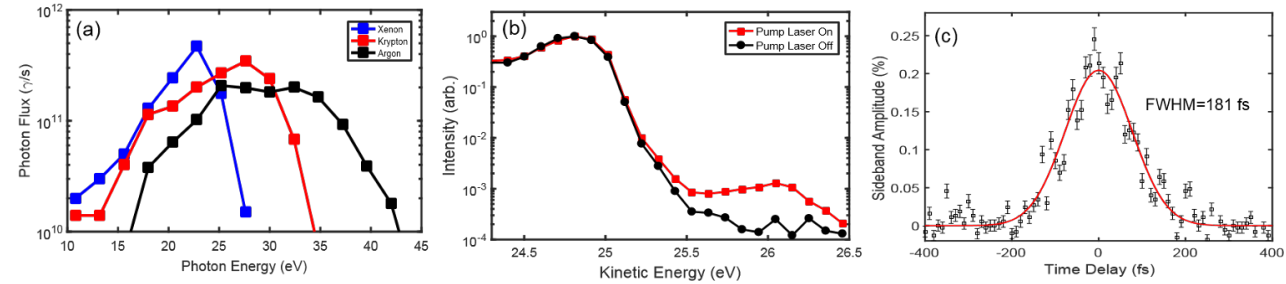

Fig. 1: (a) Typical photon flux delivered to the sample. A flux greater than $10^{11} \mathrm{photons} / \mathrm{s}$ in a single harmonic is available from $18 \mathrm{eV}$ to $37.2 \mathrm{eV}$. (b) LAPE on Au (111) surface with a pump laser with a peak intensity of $1.3 \times 10^{9} \mathrm{~W} / \mathrm{cm}^{2}$ (red) and with the pump laser off (black). (c) LAPE amplitude measured at $26 \mathrm{eV}$ kinetic energy as a function of pump laser-XUV time delay.

photoemission from the pump produces at least one order of magnitude less electrons at the sample than the XUV probe. Figure 1 (c) shows the amplitude of the sideband at $26 \mathrm{eV}$ as the pump/probe delay is varied. The data were accumulated in 10 minutes. A gaussian fit to the cross-correlation gives a FWHM of 181 fs. Taking the lowest possible value of the laser pulse duration $155 \mathrm{fs}$ then gives a conservative upper limit for the XUV pulse duration at the sample of 93 fs.

\section{Conclusion}

With our high repetition rate tunable XUV source, LAPE signals with low pump fluence are observed. Importantly, LAPE signals mimic the response of weakly excited samples. When combined with modern efficient electron energy analyzers [8], the current light source will enable studies of photoexcited surfaces with unprecedented detail. 


\section{References}

1. S. Hellmann, K. Rossnagel, B. M. Marczynski, L. Kipp," Vacuum space-charge effects in solid-state photoemission", Phys. Rev. B, 79, 12, (2009).

2. M. Plotzing, R. Adam, C. Weier, L. Plucinski, S. Eich, S. Emmerich, M. Rollinger, M. Aeschlimann, S. Mathias, C. M. Schneider, "Spin-resolved photoelectron spectroscopy using femtosecond extreme ultraviolet light pulses from high-order harmonic generation”, Rev. Sci. Instrum, 87, 043903, (2016).

3. C. Corder, P. Zhao, J. Bakalis, X. L. Li, M. Kershis, A. Muraca, M. G. White, and T. K. Allison, "Ultrafast extreme ultraviolet photoemission without space charge", submitted to Arxiv.

4. C. Corder, P. Zhao, X. L. Li, M. D. Kershis, M. G. White, and T. K. Allison," Development of a tunable high repetition rate XUV source for time-resolved photoemission studies of ultrafast dynamics at surfaces", Proceedings of SPIE, (2018).

5. X. L. Li, M. A. R. Reber, C. Corder, Y. Chen, P. Zhao, T. K. Allison,” High power ultrafast $\mathrm{Yb}$ :fiber laser frequency combs using commercially available components and basic fiber tools", Rev. Sci. Instrum, 87, 093114, (2016).

6. F. Frassetto, C. Cacho, C. A. Froud, I.C. Edmund Turcu, P. Villoresi, W. A. Bryan, E. Springate, L Poletto, "Single-grating monochromator for extreme-ultraviolet ultrashort pulses", Opt. Express, 19, 19169, (2011).

7. L. Miaja-Avila, C. Lei, M. Aeschlimann, J. L. Gland, M. M. Murnane, H. C. Kapteyn, and G. Saathoff "Laser-Assisted Photoelectric Effect from Surfaces," Phys. Rev. Lett. 97, 113604, (2006).

8. G. Schonhense, K. Medjanik, H. Elmers, "Space-, Time- and Spin-Resolved Photoemission,” J. Electron." Spectrosc. Relat., 200, 94, (2015). 